Review

\title{
Future Directions for Monitoring Treatment Response in Colorectal Cancer
}

\author{
Avery S. Walker ${ }^{1}$, Nathan P. Zwintscher ${ }^{1}$, Eric K. Johnson ${ }^{1}$, Justin A. Maykel2 ${ }^{2}$ Alexander Stojadinovic ${ }^{3}$, \\ Aviram Nissan ${ }^{4}$, Itzhak Avital ${ }^{5}$, Björn LDM Brücher ${ }^{5}$, Scott R. Steele ${ }^{1 凶}$ \\ 1. Department of Surgery, Madigan Army Medical Center, 9040 Fitzsimmons Dr., Fort Lewis, WA, USA \\ 2. University of Massachusetts Memorial Medical Center, Worcester, MA, USA \\ 3. Department of Surgery, Division of Surgical Oncology, Walter Reed National Military Medical Center, Bethesda, MD, USA \\ 4. Department of Surgery, Hadassah-Hebrew University Medical Center, Jerusalem, Israel \\ 5. Bon Secours Cancer Institute, Richmond, VA, USA
}

$\square$ Corresponding author: Scott R. Steele, MD, Department of Surgery, Madigan Army Medical Center, 9040-A Fitzsimmons Avenue, Tacoma, Washington 98431, 253/968-2200. email: harkersteele@mac.com

(c) Ivyspring International Publisher. This is an open-access article distributed under the terms of the Creative Commons License (http://creativecommons.org/ licenses/by-nc-nd/3.0/). Reproduction is permitted for personal, noncommercial use, provided that the article is in whole, unmodified, and properly cited.

Received: 2013.1I.0I; Accepted: 2013.1 I.25; Published: 2014.0I.05

\begin{abstract}
Treatment of advanced colon and rectal cancer has significantly evolved with the introduction of neoadjuvant chemoradiation therapy so much that, along with more effective chemotherapy regimens, surgery has been considered unnecessary among some institutions for select patients. The tumor response to these treatments has also improved and ultimately has been shown to have a direct effect on prognosis. Yet, the best way to monitor that response, whether clinically, radiologically, or with laboratory findings, remains controversial. The authors' aim is to briefly review the options available and, more importantly, examine emerging and future options to assist in monitoring treatment response in cases of locally advanced rectal cancer and metastatic colon cancer.
\end{abstract}

Key words: Colorectal cancer, response, biomarkers, watch and wait, imaging modalities, surgical timing

\section{Introduction}

In 2012, more than 140,000 people in the United States were diagnosed with colorectal cancer, making it the third most common cancer in both men and women. ${ }^{1}$ Despite multiple efforts aimed at early detection through screening, an estimated 50,000 deaths will occur during 2013 alone, making it the second leading cause of cancer-related deaths among men and women in the United States. Most patients will present with localized disease amenable to curative surgical resection, however, approximately $20 \%$ of patients will still present with distant metastases. While surgery remains the backbone of therapy for both colon and rectal cancer, a multidisciplinary approach has become the hallmark of treatment. Patients, who in previous years would have been precluded from curative resection based on their disease burden at presentation, may now undergo neoadjuvant therapy that results in down-staging in select circumstances. Monitoring the tumor response to therapy is therefore vital in selecting those best suited for surgery to ensure optimal outcomes.

The use of neoadjuvant chemoradiation in patients with Stage II and III rectal cancer can result in a pathologic complete response (pCR) in as many as $25 \%$ of individuals. ${ }^{2}$ Demonstrating this response preoperatively can be challenging, and selection of patients suitable for non-operative management is controversial. Ideally we could tailor the treatment approach for each patient based on their individual tumor's unique response to therapy. ${ }^{3}$ This could also 
help guide the use of surgery, chemotherapy, and radiation. In small cohorts, this is already being performed, as radical surgery in patients undergoing long course chemoradiation therapy (CRT) with evidence of a complete response is omitted in select patients. $^{3}$

Additionally, a tumor's treatment response provides significant prognostic information, as tumor downstaging following chemoradiation therapy, along with the final disease stage, is an important predictor of survival. ${ }^{4}$ Furthermore, in resectable patients, pre-operative chemoradiation therapy may result in the ability to undergo a sphincter-sparing operation and may increase the $\mathrm{R} 0$ resection rate. Neoadjuvant chemotherapy in those with liver metastases may also facilitate resection in those initially considered unresectable. All of these have the potential to improve the postoperative morbidity and mortality, decrease sexual and urinary dysfunction, as well as reduce the requirement for permanent stomas in a significant number of patients. ${ }^{4}$

In order for this to be possible, however, we must ensure an accurate assessment of the treatment response. It would be a tragedy to mistakenly declare a complete response to neoadjuvant chemoradiation therapy (CRT) and avoid a major surgical resection if potentially surgically curable disease resulted in recurrence and death. On the other hand, to perform an aggressive surgical resection on a liver metastasis, or remove an asymptomatic primary when other metastases are present that were felt to have completely responded seems duly unnecessary. Therefore, the future of these treatments, and treatment strategies, will depend on new modalities to help accurately identify how each tumor responds to therapy. The aim of this article is to inform readers of novel and evolving concepts regarding tumor response and of ways to approach the complete responder.

\section{Defining Tumor Response}

While several definitions vary in the literature, at its most basic assessment, response may be classified as complete, incomplete, or non-response. In 2000 the Response Evaluation Criteria in Solid Tumors (RECIST) guidelines were created, and subsequently updated in 2009 (Table 1). Clear definition of response criteria aids in communication as well as reporting of outcomes. These guidelines define a complete response as the disappearance of all non-target lesions and normalization of tumor marker level. ${ }^{5} \mathrm{~A}$ target lesion is defined as $\geq 1$ measurable lesion present at baseline, up to a maximum of five lesions total, representative of all involved organs, based on size. Non-target lesions represent all other lesions (or sites of disease) including pathological lymph nodes. An incomplete response or stable disease is defined as the persistence of one or more non-target lesion(s) and/or the maintenance of tumor marker level above the normal limits. ${ }^{5}$ In a study by Saur et al. in 2004, preoperative chemoradiotherapy resulted in a partial, or incomplete, response in approximately $89 \%$ of patients with rectal cancer. They also showed that after preoperative chemoradiotherapy there was a significant shift toward earlier TNM stages (i.e., downstaging), reduced rates of local failure, and decreased toxic effects. Also among patients with tumors judged by the surgeon to require an abdominoperineal excision, the rate of sphincter-preserving surgery was more than doubled after preoperative chemoradiotherapy. However, this did not result in an increase in overall survival. ${ }^{6}$

Table I. Revised RECIST guideline (version I.I)62

\begin{tabular}{ll}
\hline Grade & Response Criteria \\
\hline Complete & $\begin{array}{l}\text { Disappearance of all target lesions. Any patholog- } \\
\text { ical lymph nodes (whether target or non-target) } \\
\text { must have reduction in short axis to }<10 \mathrm{~mm} .\end{array}$ \\
$\begin{array}{l}\text { Partial Re- } \\
\text { sponse }\end{array}$ & $\begin{array}{l}\text { At least a 30\% decrease in the sum of diameters of } \\
\text { target lesions, taking as reference the baseline sum } \\
\text { diameters. }\end{array}$ \\
Progressive & $\begin{array}{l}\text { At least a 20\% increase in the sum of diameters of } \\
\text { target lesions, the appearance of one or more new }\end{array}$ \\
Disease & $\begin{array}{l}\text { lesions is also considered progression } \\
\text { Stable Disease } \\
\text { Neither sufficient shrinkage to qualify for partial } \\
\text { response nor sufficient increase to qualify for pro- } \\
\text { gressive disease }\end{array}$ \\
\hline
\end{tabular}

The ultimate goal is to avoid surgical resection and its associated morbidity, which necessitates complete resolution of the viable tumor. However, until we are able to better delineate who are appropriate candidates, tumor response is more often further characterized by histological evaluation. While this obviously requires a surgical resection, it is the most accurate method available. A pCR is the ideal response for all tumors following neoadjuvant CRT. Quah and colleagues define a pathological complete response by the absence of any viable tumor cell on the resected specimen, irrespective of the proportions of necrosis and fibrosis. ${ }^{4}$ Another commonly accepted definition of $\mathrm{pCR}$ is the Dworak tumor response grading system, which defines a complete pathologic response as the absence of residual cancer cells in the resected specimen after a radical resection (Table 2). ${ }^{7}$ Unfortunately, to gauge the pathological response, the required operation may carry significant quality of life consequences for the patient, along with a debatable oncological benefit when no tumor cells are identified. 
Table 2. The Dworak Tumor response grading system ${ }^{7}$

\begin{tabular}{|c|c|}
\hline Grade & Definition \\
\hline Grade 0 & No response \\
\hline Grade 1 & $\begin{array}{l}\text { Minimal response (dominant tumor mass with obvious } \\
\text { fibrosis, vasculopathy) }\end{array}$ \\
\hline Grade 2 & $\begin{array}{l}\text { Moderate response (dominant fibrotic changes with a } \\
\text { few easy-to-find tumor cells or groups) }\end{array}$ \\
\hline Grade 3 & $\begin{array}{l}\text { Near complete response (few microscopically diffi- } \\
\text { cult-to-find tumor cells in fibrotic tissue with or without } \\
\text { mucous substance) }\end{array}$ \\
\hline Grade 4 & $\begin{array}{l}\text { Complete response (no tumor cells, only fibrotic mass or } \\
\text { acellular mucin pools) }\end{array}$ \\
\hline
\end{tabular}

Ideally, accurately predicting the absence of residual tumor cells following chemo and/or radiation therapy would be possible without the need to undergo a major operative procedure. Hence the need to define the clinical response to treatment, and more importantly, what entails a complete clinical response (cCR) (Figure 1). Habr Gama and associates define a complete clinical response (cCR) as the absence of residual tumor within the context of multiple clinical evaluations to include rigid proctoscopy, digital rectal examination (DRE), presence of an ulcer at the site of the lesion, or any lesion or residual calcification at the site of the initially detected metastases on all imaging modalities. Standardization of the definition of CCR and PCR is crucial for the development and understanding of response assessment in the management of colon and rectal cancer. The revised RECIST guidelines are currently the closest standardized and accepted response criteria available.

Unfortunately, a cCR does not necessarily equate to pCR. Chari and associates found 22 of 43 $(51 \%)$ patients who underwent preoperative chemoradiation therapy for rectal cancer to have a complete clinical response, however, only 11 of those 22 patients had a complete pathological response. ${ }^{8}$ Seong et

al. reported a complete clinical response rate of $23.8 \%$ in patients with primary unresectable rectal cancer receiving preoperative chemoradiation; however, after resection, only $9.5 \%$ had a complete pathologic response. ${ }^{9}$ Nair and colleagues demonstrated a similar discrepancy between complete clinical responders (70\%) and complete pathologic responders $(57 \%)$ in 2008. ${ }^{10}$ All three studies mentioned here used a combination of proctoscopy, endorectal ultrasound (ERUS), computed tomography, and biopsy information to determine response; yet, there was no standard definition of response or how to determine a response amongst the studies. More recently, Issa and associates demonstrated a 39\% complete clinical response in 174 patients with rectal cancer receiving CRT. Of the 38 patients that underwent a local excision after CRT, 8 showed evidence of residual disease, which comprised $25 \%$ of the patients who were initially identified as complete clinical responders. ${ }^{11}$ These studies (Table 3) plainly show a disconnect between those identified as a complete clinical responder and who really has a complete pathological response. The evolving definition and ability to identify the clinical responder is the crux of this review and the current dilemma.

Table 3. Studies of Complete Clinical Responders and Pathologic Complete Responders

\begin{tabular}{llll}
\hline Author & Year & cCR \% & pCR \% \\
\hline Issa & 2012 & 39 & 25 \\
Nyasavajjala & 2009 & 30 & 10 \\
Nair & 2008 & 70 & 57 \\
Chari & 2005 & 51 & 27 \\
Hiotis & 2002 & 75 & 25 \\
Seong & 2001 & 23.8 & 9.5 \\
\hline
\end{tabular}

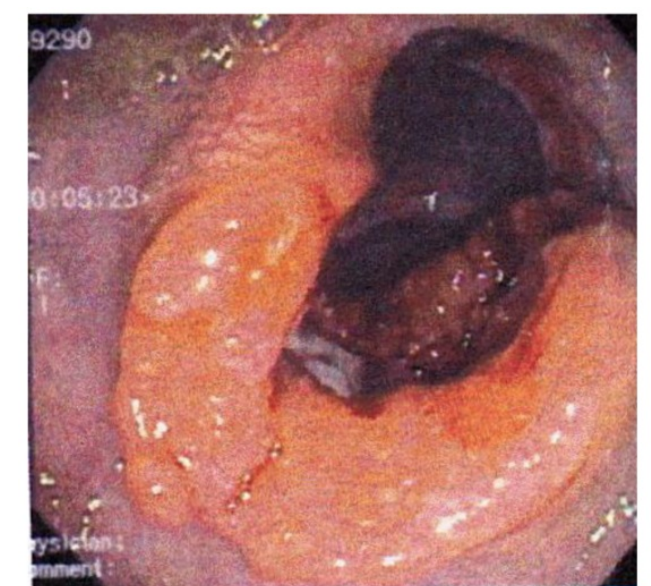

(A)

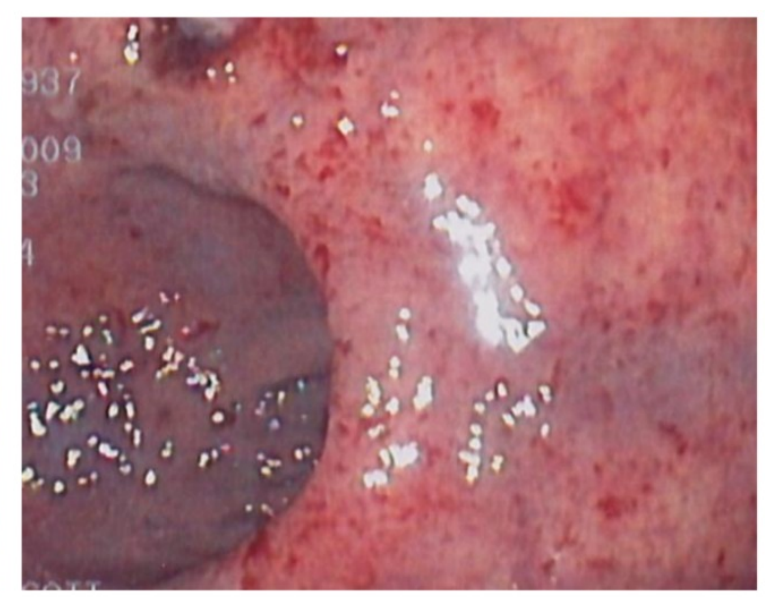

(B)

Figure I. (A) Pre and (B) Post-Neoadjuvant ChemoradiationTherapy (CRT) Demonstrating a Complete Clinical Response (cCR). Courtesy of Scott R. Steele, MD 


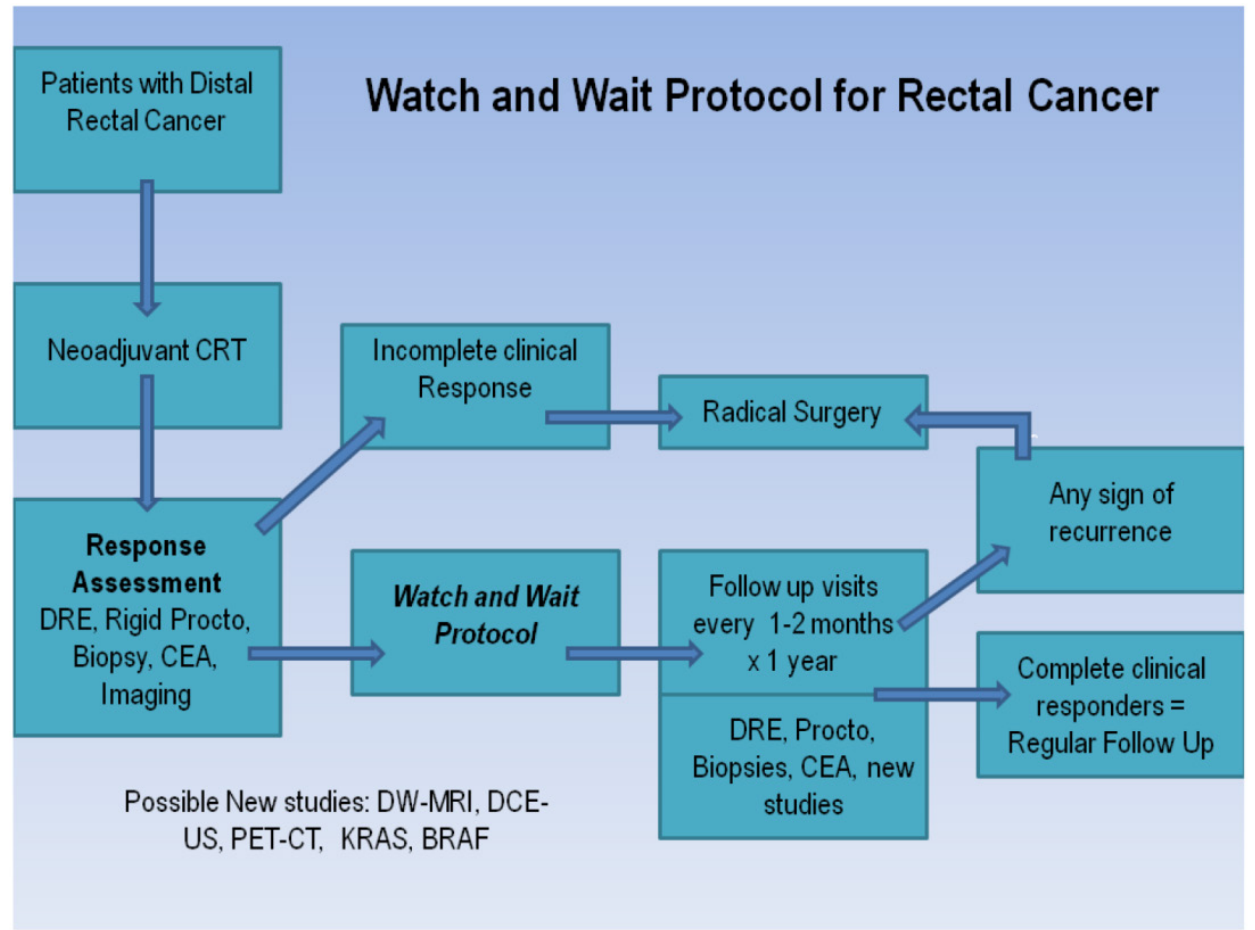

Figure 2. Watch and Wait Protocol ${ }^{12}$

Table 4. Tools to Detect Tumor Responses

\begin{tabular}{lll}
\hline Clinical & Labs & Radiological \\
\hline Digital Rectal Exam & CEA & PET-CT \\
Rigid Proctoscopy & KRAS/BRAF & Diffusion Weighted MRI \\
Wall Pliability & CpG- IMP & ERUS \\
Biopsy & & DCE-US \\
\hline
\end{tabular}

CEA - Carcinoembryonic antigen, CpG IMP - Island Methylation Phenotype, ERUS - Endorectal Ultrasound, PET CT - Positron emission tomography - computed tomography, DCE- US - Dynamic contrast-enhanced ultrasonography

\section{Identifying the Extent of Tumor Response}

Factors related to tumor response are multifactorial, and typically rely on the specific treatment regimen, timing of chemotherapy, and likely most importantly, the individual patient's tumor biology. Regardless, in order to change the paradigm of colorectal cancer therapy going forward, we must be able to both accurately predict the degree of response, as well as have uniform definitions across all disciplines. Habr-Gama and colleagues have devised a concise and easy-to-follow algorithm to help distinguish complete from incomplete responders to neoadjuvant chemoradiation therapies for rectal cancer based on clinical, radiological, and endoscopic findings (Figure 2). The assessment includes a digital rectal examination (DRE), rigid proctoscopy, biopsy of the suspicious lesion (if present), carcinoembryonic antigen (CEA) level, and one or more radiological studies (abdominal/pelvic CT, PET-CT, or MRI). ${ }^{12}$ On DRE, whenever a tumor cannot be felt or seen, patients are considered complete responders. With proctoscopy, whitening of the mucosa in an area of the rectal wall may be frequently observed in patients with a complete response. During manual air insufflation, a subtle loss of the pliability of the rectal wall harboring the scar can often be a sign of an incomplete clinical responder. In contrast, a residual superficial or deep ulceration with or without a necrotic center, or a palpable nodule in the presence of mucosal integrity are all concerning for a recurrence and should warrant a more invasive evaluation to include a full-thickness transanal excision or transanal endoscopic microsurgery (TEM) where available. Caution must be taken if this approach is used, as demonstrated in the small study by Perez and colleagues, as a $15 \%$ local failure rate has been reported after an R0 transanal endoscopic microsurgery for patients with ypT0-2 lesions. ${ }^{13}$ While these approaches may provide definitive information regarding the patient's tumor response when only subtle positive signs of residual disease are present, they may not guarantee curative surgery in favor of standard total mesorectal excision.

The Habr-Gama group is the only group at this time to demonstrate a clear and concise protocol to follow patients who show clinical evidence of a complete response. For stage 0 rectal cancer patients, they observed 265 patients who underwent neoadjuvant chemoradiation therapy and compared the outcomes of patients who were treated nonoperatively and op- 
eratively thereafter. Five-year overall and disease-free survival rates were $88 \%$ and $83 \%$, respectively, in the resection group and $100 \%$ and $92 \%$ in the observation group. These findings are truly unique in that no other group has been able to duplicate these findings. Hughes and colleagues studied 58 patients with rectal cancer post-CRT who did not proceed to surgery; 10 $(17 \%)$ of these had a clinical complete response (cCR). Six of the 10 developed intra-pelvic recurrent disease $(60 \%)$ and the median time to local disease progression was 20 months (range 10-80 months). ${ }^{14}$ The reasons for not proceeding with surgery in these 10 patients were patient choice in three patients and co-morbidities in seven patients. Nyasavajjala and associates found only $10 \%$ of patients demonstrated a pathological complete response to neoadjuvant CRT (Table 3). ${ }^{15}$ These studies and others clearly show how the difficulties of accurately predicting a complete pathological response limit the use of clinical response alone as an end point for determining future management.

Part of the issue in accurately determining a complete response clinically is that both DRE and post-CRT MR scanning are unreliable for distinguishing fibrosis from small islands of tumor tissue, yielding a low positive predictive value. A retrospective study by Hiotis and associates, comparing the clinical assessment and final pathological findings in a group of patients with rectal cancer, showed that the pathologic complete response rate among those determined to have a complete clinical response was only $25 \%$. While a cCR was a significant predictive factor for pathologic complete response, the majority of the patients studied had persistent foci of tumor that were not detectable on preoperative examination or proctoscopy..$^{16}$ Further concerns were raised regarding the clinical assessment of response by the report from Hayden and colleagues, where they introduced the notion of "tumor scatter," defined as the 1) presence of cancer cells outside of the visible ulcer or 2) microscopic tumor cells present in the absence of a visible ulcer. Residual tumor was identified outside the visible ulcer or in the absence of an ulcer in $49 \%$ of patients, including some up to $4 \mathrm{~cm}$ away from the primary. Moreover, a small number demonstrated $3 \mathrm{~cm}$ of distal spread beyond the ulcer bed. ${ }^{17}$ Based on the available evidence, the authors felt that clinical examination and determination of response is not an appropriate predictor of pathologic response at this time-corroborated by several other series. In the absence of validated imaging technologies, gene expression techniques, or laboratory findings, we are left with clinical examination as the only modality to assess response in rectal cancer. However, to use this solely as the basis to avoid surgery at the present time, we feel this cannot currently be recommended outside the setting of a clinical trial.

\section{Laboratory Evaluation}

Routine laboratory examinations including complete blood cell counts, liver function tests, coagulation profiles, and chemistry panels are performed during the evaluation and follow-up care of patients with colorectal cancer. However, none of these basic laboratory tests have yet to demonstrate a role regarding an accurate measurement of treatment response. Other laboratory findings continue to have an undefined role in this capacity. Most scientific information is in the preclinical stages, however, there is promise that one or more of the following laboratory findings may play a larger role in determining treatment guidance as well as treatment response.

\section{CEA}

Serum carcinoembryonic antigen (CEA) is still the most widely used tumor marker in patients with colorectal cancer. Pretreatment CEA levels are useful for prediction of prognosis, and postoperative serial assays of CEA level provide an opportunity for early detection of recurrent disease. ${ }^{18}$ Reduction of the CEA level after radical resection has been associated with improved survival in rectal cancer. ${ }^{19}$ CEA levels before and after neoadjuvant CRT may also be useful when determining complete vs. incomplete responders. CEA levels $\geq 5.0 \mathrm{ng} / \mathrm{mL}$ have an adverse impact on survival that is independent of tumor stage. ${ }^{20}$ In a series of 17,910 patients diagnosed with colon cancer of any stage, an elevated preoperative CEA level was associated with a significantly increased risk of overall mortality (hazard ratio for death 1.60, 95\% CI 1.46 to 1.76$).^{21}$

With regard to its role in assessing tumor response to therapy, CEA has limited data at present. A retrospective analysis of 109 patients undergoing neoadjuvant therapy identified a cutoff value for $\mathrm{CEA}<2.7 \mathrm{ng} / \mathrm{ml}$ at 4 weeks from $\mathrm{RT}$ completion to be a statistically significant marker of tumor regression (OR 0.166, 95\% CI 0.057-0.484, $P=.001$ ). ${ }^{22}$ The Habr-Gama group has found and incorporated a low post-CRT CEA level of $<0.5 \mathrm{ng} / \mathrm{dL}$ to be a significant predictor of a complete clinical response and improved overall and disease-free survival, regardless of initial CEA levels into their current algorithm for rectal cancer response.

\section{KRAS/BRAF}

Mutations in KRAS codons 12, 13, or 61 are common in colorectal tumors and produce a constitutively active ras protein, leading in turn to EGFR-independent activation of the mito- 
gen-activated protein kinase pathway (MAPK) pathway. ${ }^{23}$ This pathway results in proliferation, adhesion, angiogenesis, migration, and survival. The BRAF gene, which encodes a downstream effector of KRAS in the MAPK pathway, is also mutated in a subset of metastatic colorectal cancers. ${ }^{24}$ BRAF mutations are strongly associated with a worse outcome in CRC patients. ${ }^{25}$ The incidence of the BRAF mutation in colorectal cancer is $\sim 8-10 \% .{ }^{26}$ Within the large phase III CRYSTAL trial, BRAF mutant tumors did poorly, regardless of the therapy they received. Evaluation of BRAF in Stages II and III colon cancers showed that BRAF mutation was a negative prognostic factor for overall survival. The randomized Fluorouracil, Oxaliplatin, and Irinotecan: Use and Sequencing (FOCUS) and CAIRO2 studies demonstrated that tumor BRAF mutation was a negative prognostic marker for overall survival in patients with metastatic CRC. ${ }^{24,} 27$ In another study conducted by Yokota et al., the BRAF mutation was shown to be an independent poor prognostic factor for survival in patients with advanced and recurrent colorectal carcinoma. As prognostic markers, KRAS and BRAF definitely seem promising, though data regarding the assessment of tumor response is evolving.

One way in which KRAS/BRAF may be considered with treatment response in mind, is that the presence or absence of a mutation can help discussions with patients to allow for a closer/longer follow-up interval as the previous studies also demonstrated a more aggressive tumor less likely to respond to standard CRT. Based on the information available, BRAF mutation testing has other potential clinical applications. Importantly, surgeons and oncologists may use the result to determine the likelihood of response to epidermal growth factor receptor inhibitor therapy, in that, lack of a BRAF mutation could provide further guidance and closer follow-up if this mutation is known prior to treatment. ${ }^{28}$.

For patients with KRAS positive cancer, it is also important going forward for us to establish whether these mutations, in addition to precluding benefit from anti- EGFR monoclonal antibody therapies, may affect the ability to benefit from other chemotherapeutic agents as well as accurately determine the prognosis independent of treatment. This information may be needed for counseling individual patients who present with such mutations. In regards to response to treatment, knowing that these markers are present may alter the follow-up evaluation on a higher acuity interval. Similarly, as this may be a marker of a more aggressive tumor that is not very responsive to standard treatment modalities, this may also allow the clinician to offer more aggressive or experimental treatments.

\section{CIMP}

$\mathrm{CpG}$ island methylation phenotype (CIMP) is DNA methylation at the cytosine base of CpG dinucleotide islands, by DNA methyltransferase enzymes. ${ }^{29}$ Within cancer cells, it has been observed that there is genome-wide hypomethylation and gene promotor hypermethylation. Hypermethylation contributes to gene silencing and genomic instability and affects tumor-suppressor genes, DNA repair, and cell-cycle control. CIMP is detected in approximately $30-40 \%$ colon cancers. Toyota and associates first described CIMP in CRC, identifying cancer-specific methylation and distinguishing it from age-specific methylation. ${ }^{30}$ Subsequently, Wiesenberger and associates performed unsupervised two-dimensional cluster analysis of DNA methylation and classified CRC into CIMP-negative or CIMP-positive cancers. ${ }^{31}$ They observed a strong relationship of CIMP cancers with BRAF mutations. DNA hypermethylation is under investigation both as a tool in colon cancer screening and a target for cancer therapy. One method of utilizing DNA methylation in CRC screening is by detecting abnormal DNA methylation from tumor cells shed in stool samples. An early study performed by Muller and associates found methylated DNA in stool samples of 13 patients with colorectal cancer and three of 13 without the disease (sensitivity 90\% [CI 56-100\%] and specificity 77\% [CI 46-95\%). ${ }^{29}$ When, and if, therapies are directed at these genotypes, the ability to detect the levels within tumor cells shed in stool may make assessing response easier and possibly provide the ability to follow the response to therapy "real-time" throughout treatment. Molecular biology will continue to provide additional information regarding tumor response to neoadjuvant CRT. The biomarkers discussed will eventually be further validated and added to regimens for tumor detection, tumor response, and treatment-based schemes.

\section{Imaging of Response}

Imaging modalities such as chest/abdominal/ pelvis CT scans and MRI are the standard recommendations for the follow-up evaluation of colon cancer treatment; however, standard radiologic assessment with CT scans, high-resolution MRI, and endorectal ultrasound have not added significant accuracy in identifying patients with complete tumor regression. The assessment of response by radiological studies to the treatment of colorectal cancer is growing quickly as technology advances. Currently, we assess response with imaging per the RECIST criteria, which was updated in 2009 (Table 1). 


\section{CT}

At present, computed tomography's (CT) use is primarily in a surveillance role, after definitive surgical therapy for patients at higher risk of recurrence, and typically for those with node-positive tumors, where the American Society of Clinical Oncology (ASCO) recommends annual CT of the chest and abdomen for 3 years to include the pelvis in rectal cancer patients. ${ }^{32} \mathrm{~A}$ systematic review of studies by Bipat and associates comparing the diagnostic performance of different imaging modalities for the detection of colorectal liver metastases, found that $\mathrm{CT}$ achieves a sensitivity of $64.7 \% .{ }^{33} \mathrm{CT}$ is currently used to demonstrate early, mass-like tumor recurrence at the surgical anastomosis due to the often largely extrinsic component of such recurrence. ${ }^{34}$ This appearance can mimic postoperative fibrosis, although fibrosis usually appears more linear without a discrete mass. Occasionally, distinction between postoperative fibrosis and recurrent tumor is not possible unless serial scans are obtained. CT findings clearly indicative of recurrent malignant disease include enlargement of a soft-tissue mass over time, enlarging regional lymphadenopathy, and invasion of contiguous structures. Local relapse in rectal carcinoma has been significantly reduced since the introduction of total mesorectal excision (TME) and radiation therapy. The sensitivity for $\mathrm{CT}$ in this scenario is reported to be $82 \% .{ }^{35}$ However, specificity is as low as $50 \%$ reflecting the difficulty in distinguishing recurrent tumor from post-operative fibrosis. ${ }^{35} \mathrm{Up}$ and coming imaging modalities may be able to differentiate this conflict.

Yet, imaging also plays a role in the assessment of tumor response as well. Evaluating for any changes in number and size of the primary or metastatic lesions is a fairly standard practice. However, as technology progresses and imaging modalities become much more sensitive, size alone may not be the only indicator of response on imaging. Chun et al. observed that in patients treated with bevacizumab for metastatic colon cancer, the most relevant aspect of tumor response is a modification of the computed tomography morphology, involving a transformation of the metastases from heterogeneous lesions with thick, irregular borders into bland, homogeneously hypodense masses with a sharp, non-enhancing interface between the tumor and adjacent liver parenchyma (Figure 3). ${ }^{36}$ Morphologic criteria correlated strongly with the percentage of residual tumor cells and also with pathologic response whereas RECIST did not. Furthermore, patients with optimal morphologic response had median overall survival of 31 months (95\% CI, 26.8-35.2 months) compared with 19 months (95\% CI, 14.6-23.4 months) with incomplete or no morphologic response $(\mathrm{P}=0.009) .{ }^{36}$ These results highlight the importance of radiological response in addition to baseline clinical factors in determining patient outcomes.
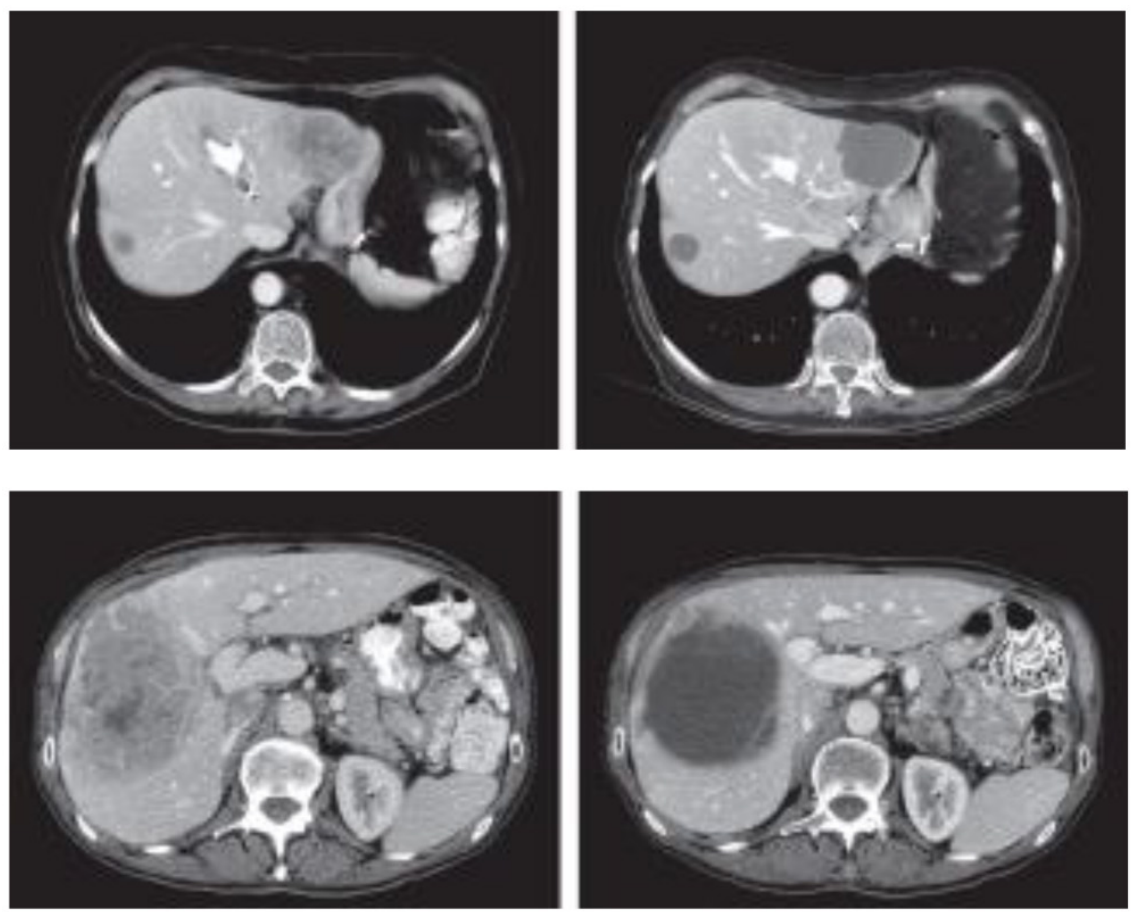

Figure 3. Transformation of the metastases from heterogeneous lesions with thick, irregular borders into bland, homogeneously hypodense masses with a sharp, non-enhancing interface between the tumor and adjacent liver parenchyma. Courtesy of Chun et al. ${ }^{36}$ 


\section{MRI}

Similar to $\mathrm{CT}$, magnetic resonance imaging (MRI) primarily is being used in a surveillance role or differentiating benign from recurrent disease, though with a higher sensitivity $(75.8 \%)$ than CT for the detection of colorectal liver metastases. ${ }^{33}$ However, the higher cost of MRI and its limited value in detecting lung metastases precludes its use over CT for routine surveillance. It is therefore reserved for more accurate staging of the liver, often when surgery or thermal ablation is being considered. Furthermore, MRI is more sensitive than CT for the detection of pelvic recurrence, where once again it may be useful in treatment planning particularly where the surgical resection plane requires definition. Tumor response detection by non contrasted MRI was demonstrated in a recent study by Ricotta et al. They showed that radiologic evaluation with MRI of metastatic colorectal cancer after two weeks of treatment was able to predict subsequent radiologic response. Tumor shrinkage $>10 \%$ represented an early indicator of clinical outcome because it is predictive of the prolongation of progression-free survival and overall survival. They found that early responding patients had a very high probability of achieving either a partial response or control of disease progression as evaluated by RECIST criteria. ${ }^{37}$

However, the use of MRI as part of routine follow-up and assessment of response has been questioned. In a study of 226 patients, MRI detected the same proportion of resectable tumors (4 of 6 ) as that diagnosed by conventional follow-up tests. ${ }^{36}$ Although two additional cases of resectable tumor were found when MRI was used in addition to conventional tests, this benefit was considered to be out- weighed by a large proportion (14\%) of false-positive tests resulting in additional cost and patient anxiety. ${ }^{36}$ More promising for assessing tumor response is Diffusion-Weighted (DW)-MRI. This functional imaging technique yields qualitative and quantitative information and provides unique insights regarding tumor cellularity, integrity of cell membranes, and microcirculation. ${ }^{38}$ Apparent diffusion coefficients (ADC), which are quantitative expressions of diffusion characteristics of tissues, tend to decrease in diffusion restricted areas, whereas diffusion signal intensity (SI), which is the qualitative parameter of diffusion, increases in those areas. ${ }^{38}$ While not quite ready for use on a widespread scale, eventually by comparing the mean ADC values of a lesion before and after treatment, this can be used as another marker of tumor response.

Lambregts et al. studied a total of 120 patients with locally advanced rectal cancer from three university hospitals who underwent chemoradiation therapy followed by standard T2 weighted-MRI and diffusion weighted imaging (DWI) (Figures 4 and 5). The sensitivity for selection of complete responders ranged from $0-40 \%$ on standard MRI versus $52-64 \%$ after the addition of DWI. They concluded that MRI+ DWI could significantly improve sensitivity for selection of complete responders. ${ }^{39}$ Furthermore, specificity is greater than $90 \%$, which indicates that the risk for underestimation of residual tumor can be brought to $<10 \%$. As an adjunct to clinical tools (i.e., DRE, endoscopy, and biopsy), the combined use of MRI +DWI seems promising to enable a more precise selection of patients eligible to undergo less invasive treatments. The current results are obviously still premature for clinical decision-making, but its promise warrants further large and prospective patient studies.
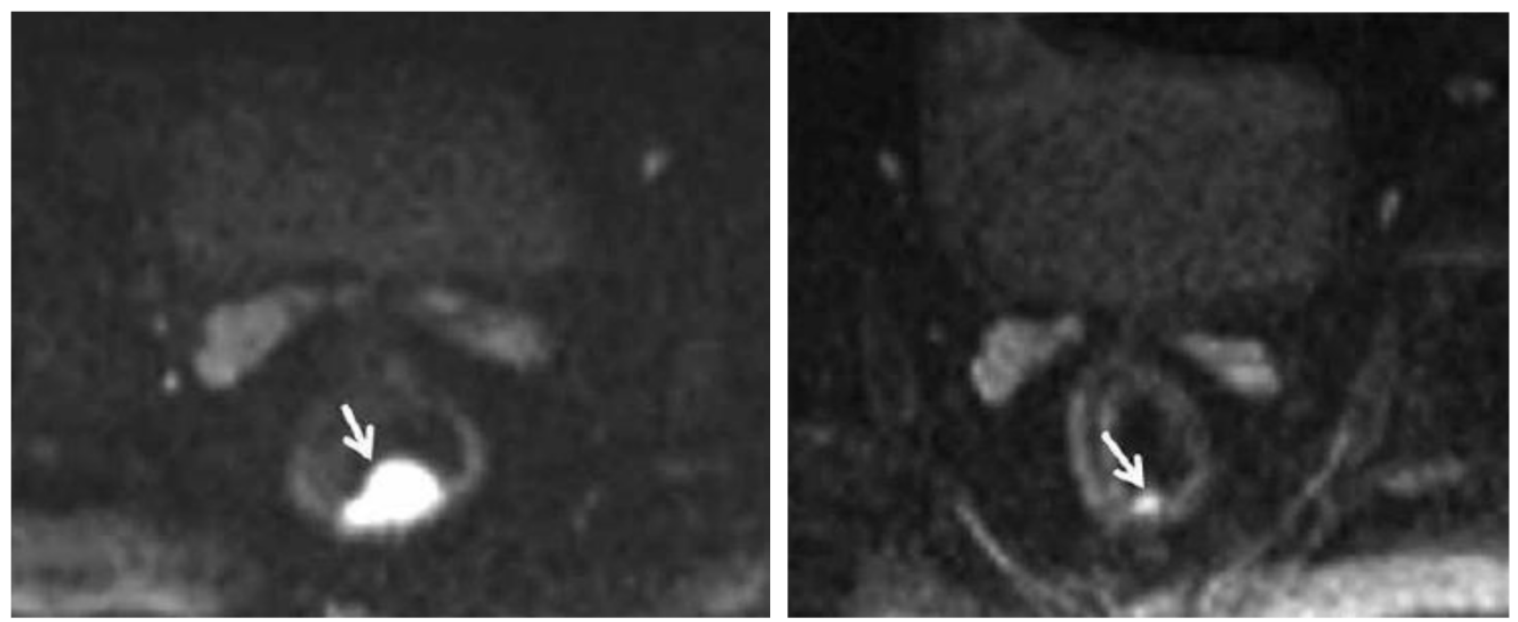

Figure 4. Pre-chemoradiation therapy (CRT) DW-MRI (left) shows a $4.8 \mathrm{cc}$, polypoid rectal tumor with very high signal intensity (arrow). Post-CRT DW-MRI (right) shows a significant decrease in tumor volume $(\mathrm{I} \mathrm{cc})$. Residual tumor with intermediate signal intensity limited to the mucosa and submucosa can be seen (arrow). Courtesy of Engin, et al. ${ }^{63}$ 

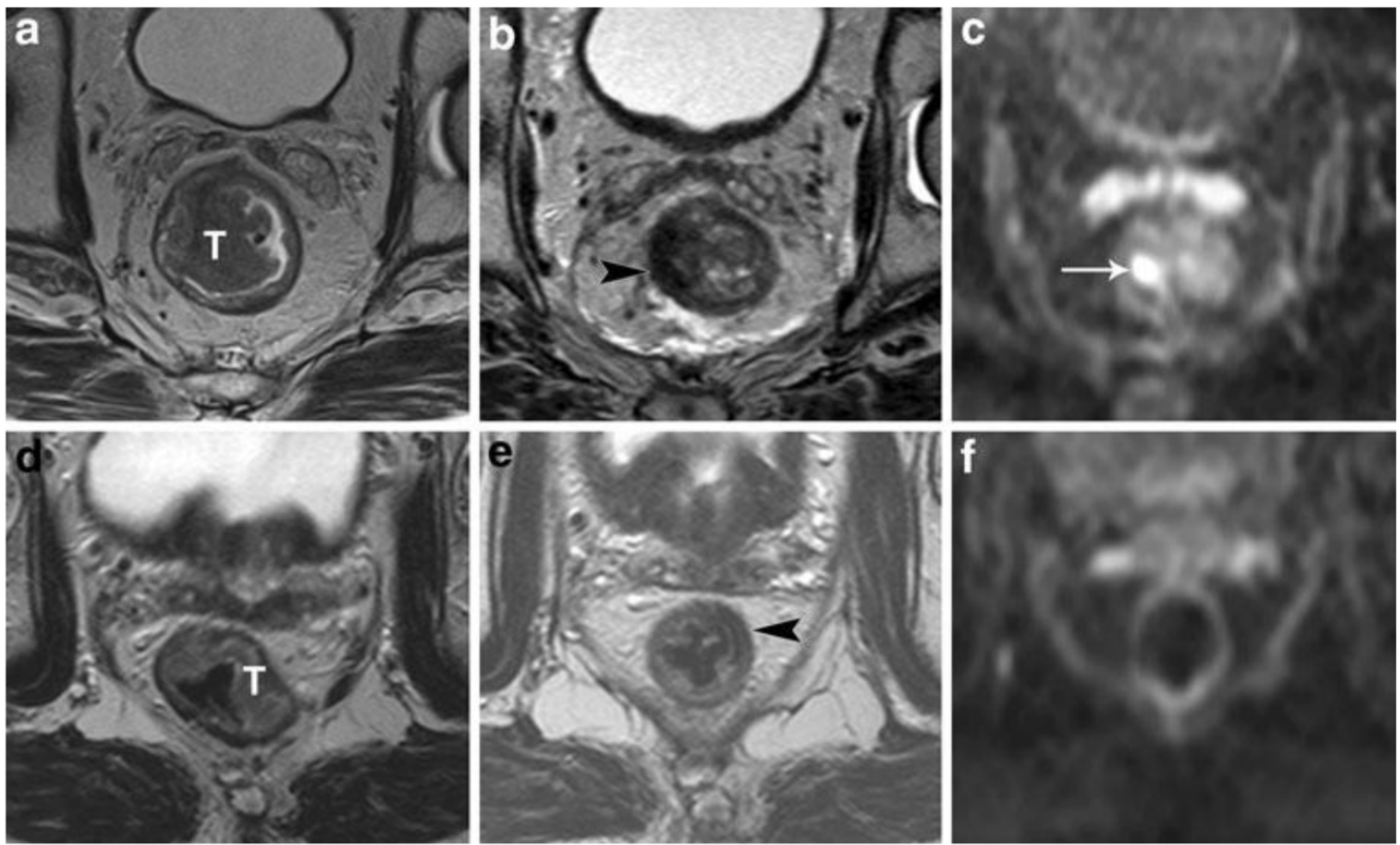

Figure 5. StandardT2-weighted images of two patients with a tumor $(\mathrm{T})$ in the rectum before $(\mathrm{a}, \mathrm{d})$ and after chemoradiation treatment (b,e). In the upper patient there is still a clear high signal intensity area on DWI (arrow in c), which was confirmed to be a PT2 residual tumor at histology. In the lower patient, no high signal is shown on DWI (f) and a complete tumor response (pT0) was confirmed at histology. Courtesy of Lambregts, et al. ${ }^{39}$

\section{PET-CT}

Radiologic studies that incorporate metabolic information in addition to standard radiologic imaging are expected to improve the overall accuracy of detecting patients who have complete tumor regression. The role of positron emission tomography integrated with computed tomography (PET-CT) regarding tumor response is not yet clearly defined. Overall, positron emission tomography with fluorodeoxyglucose (FDG-PET) is significantly more sensitive $(94.6 \%)$ than CT or unenhanced magnetic resonance imaging (MRI) for the detection of liver metastases on a per patient basis. ${ }^{33}$ However, the high cost and lower availability of PET preclude the use of this modality for routine surveillance-a separate issue from predicting tumor response.

FDG-PET is also of value and presently used in the investigation of patients with raised tumor markers and negative conventional imaging. The positive yield of FDG-PET in this situation ranges between 38 and $77 \% .{ }^{40}$ This can be potentially expanded to predicting tumor response following CRT. In 2013, Perez and colleagues reported their results of a prospective randomized trial in which PET-CT is used to evaluate the inguinal nodes in patients who received neoadjuvant chemoradiation therapy for low rectal cancer. ${ }^{41}$ Patients were assessed for tumor response at 12 weeks after the completion of CRT by a single colorectal surgeon who used clinical and endoscopic approaches identical to those used at the initial assessment.
Overall, PET-CT findings compared with clinical and pathologic findings and resulted in a sensitivity of $93 \%$, specificity of $53 \%$, a negative predictive value of $73 \%$, a positive predictive value of $87 \%$, and an overall accuracy of $85 \%$ for the detection of persistent residual cancer (i.e. an incomplete response) ${ }^{41}$ They concluded that the combination of clinical and PET-CT findings provide a safe and appropriate selection of patients who may attain a CCR after neoadjuvant chemotherapy. As previously stated, adding a combination of imaging modalities to the clinical aspect of detecting tumor response is of extreme importance. The addition of DW-MRI along with improvements in functional CT scans is very promising with regards to attaining the ideal regimen to detect the complete clinical and pathologic responder.

\section{Endorectal Ultrasound / Dynamic Con- trast Ultrasound}

Endorectal ultrasound (ERUS) is currently the most widely used and effective diagnostic modality in the primary assessment and staging of rectal cancer. Its accuracy in numerous trials and meta-analyses ranges from 80 to $95 \%$ for T-staging and 70 to $75 \%$ for $\mathrm{N}$-staging. ${ }^{42}$ In experienced hands, ERUS can accurately measure the size, circumference, and distance of the tumor from various anatomic landmarks (e.g., anal verge, anorectal line). It is capable of examining the anal sphincters for defects as well as tumor infiltration, allowing the surgeon to decide whether a 
sphincter-sparing resection is safe or feasible. ${ }^{43}$ ERUS can also demonstrate the relationship of tumor to the pelvic peritoneal reflection (PPR), information that will help the clinician determine whether local excision is possible or preoperative chemoradiation necessary. ${ }^{4}$

The ability of ERUS to accurately evaluate tumor response to neoadjuvant chemoradiation prior to surgical resection is hampered primarily by the effects of the chemoradiation itself: tumor necrosis, fibrosis, and peritumoral inflammation caused by therapy can significantly compromise staging accuracy. These reactions may all appear sonographically indistinguishable from residual tumor, obscuring differentiation of the five layers of the rectal wall and resulting in overstaging. In a study by Gavioli and associates, twenty-nine patients were subjected to endorectal ultrasound before and after preoperative radiotherapy. The patients then underwent a surgical resection followed by histological examination and comparison to the ERUS results. ${ }^{45}$ Morphologically and quantitatively, post-radiation endorectal ultrasound showed the reappearance of anatomic cleavage planes, a considerable shrinkage of the tumor; and in low rectal tumors, an increase in the distance from the anorectal ring in more than 50 percent of the cases. Histologic examination showed that in 28 out of 29 cases, fibrosis was the most dominant component of the irradiated lesions, varying by more than 50 to 100 percent of the lesion. A comparison of post-radiation ERUS with histopathology revealed that fibrosis became the morphologic basis of ultrasound images; therefore, after radiotherapy, what endorectal ultrasound staged was no longer the tumor, but the extent of fibrosis in the rectal wall. ${ }^{45}$ Radovanovic and associates looked at the accuracy of staging locally advanced rectal cancer after preoperative chemoradiation using ERUS. They found the accuracy of ERUS for T stage after chemoradiation was $75 \%$ in 33 of 44 patients. Overstaging occurred in $18 \%$ of patients, and $7 \%$ were understaged (Figure 6). Interestingly, five patients $(11.4 \%)$ had a complete histological regression and one of these patients was staged correctly, while the other four were over staged. In the detection of perirectal lymph node metastases, ERUS was accurate in $68 \%$ of patients. ${ }^{46}$ Overall, it appears that ERUS has an important role in restaging rectal cancer after neoadjuvant treatment; however, based on the results above and other studies reporting similar results, one must take into consideration some specific changes in rectal wall and surrounding structures that can lead to false staging. Furthermore, it does not appear that it has high enough accuracy to completely differentiate a patient with a complete response from those with residual tumor.

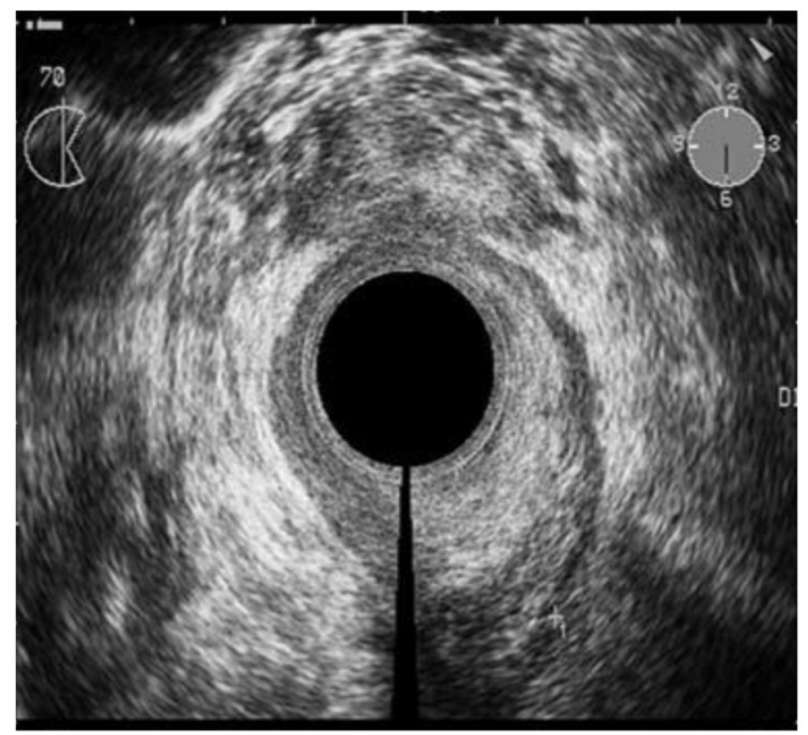

Figure 6. Hypoechoic zone staged as T2 cancer by ERUS. Pathologic analysis of the specimen showed complete response to neoadjuvant treatment (pT0). Courtesy of Radovanovic et al. ${ }^{46}$

Looking to the future, three-dimensional ERUS (3D-ERUS) enhances the understanding of the spatial relations of rectal tumors resulting in improved staging and assessment of resectability. ${ }^{47}$ The accuracy of 3D-ERUS, 2D-ERUS and CT for assessing the depth of tumor infiltration was $78 \%, 69 \%$, and $57 \%$, respectively, while for evaluating nodal involvement it was $65 \%, 56 \%$, and $53 \%$, respectively. 48 Such 3D images have proved a better definition of the mesorectal margins, thus overcoming one of the limits of two-dimensional ultrasound scans. ${ }^{49}$ 3D-ERUS could assist endoscopic mucosal resections of early tumors for a safer and more effective procedure and accurate volumetric measurements with this technique. Ideally, it could be used to better predict response after chemoradiation therapy for rectal lesions by improving the detection and level of tumor invasion as well as providing a more accurate assessment of lymph nodes. Yet in order for this to occur, additional information such the appearance and echogencity of small and micro-metastases via ultrasound will have to be delineated.

One promising advance based on US technology as it applies to assessing tumor response is the emerging use of dynamic contrast-enhanced ultrasonography (DCE-US), a new functional technique that enables a quantitative assessment of solid tumor perfusion. ${ }^{50}$ Reduction in tumor vascularization can be detected in responders after only 1 or 2 weeks, and trans-abdominal DCE-US has therefore been proposed as an alternative method for measuring early response to treatment that could be predictive of long-term survival in metastatic colon cancer, as well as non-metastatic colon cancer. In a study by Onji and 
associates, they found a statistically significant difference in the microvascular structure between colon cancer and acute inflammation. ${ }^{51}$ The microvascular structure in cases of colon cancer was irregular and characterized by large vessels (Figure 7). In addition, both abnormalities were found in a majority $(58.8 \%)$ of cases of colon cancer but in none of the cases of acute inflammation. ${ }^{51}$ Thus, it was possible to distinguish colon cancer from acute inflammation by evaluating the microvascular structure ultrasonographically. This technique has the potential for use in tumor response monitoring that may ultimately be less expensive and less invasive. At present, the evidence to support its use is too limited.

\section{Response to Treatment: Metastases}

The liver is the most common site of CRC metastasis and upwards of $25 \%$ of patients with colorectal cancer will have liver metastases at the time of initial diagnosis.52 For those with resectable disease, liver metastasectomy can be performed before, after, or simultaneously with the primary tumor resection, although the addition of neoadjuvant chemotherapy with surgery in otherwise acceptable liver resection candidates has been shown to mildly increase progression-free survival by $9 \%$ and can be given some consideration. ${ }^{53}$ It is important to remember that overall survival after liver resection is not related to the response to chemotherapy, but is largely a func-

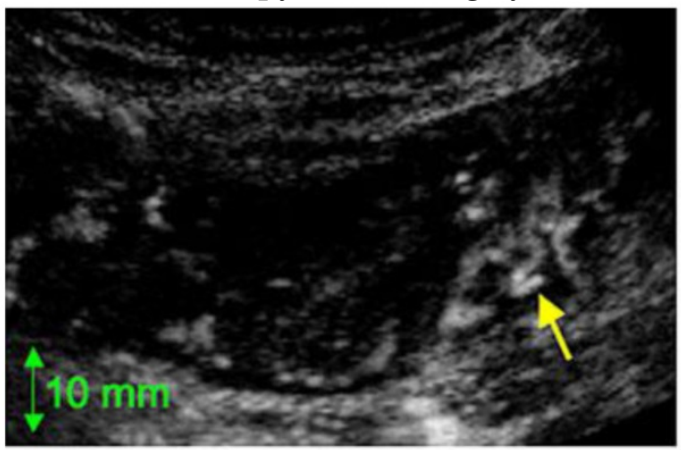

a Irregular structure

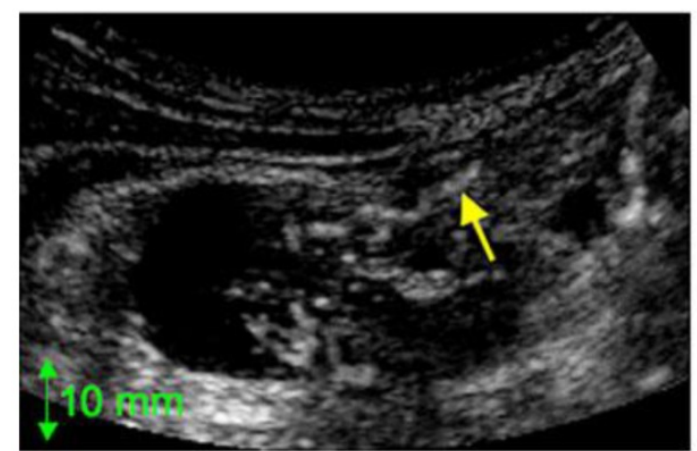

c Vessel diameter $\geq \mathbf{2} \mathbf{m m}$ tion of the original staging at time of initial diagnosis with a significantly higher five-year survival for those with Stage 1 or 2 disease versus those with Stage 3 or 4 disease $(83.9 \%$ vs. $35.7 \%) .{ }^{54}$

For hepatic disease that is initially unresectable, it is important to obtain follow-up imaging to assess for response after chemotherapy, as unresectable disease can ultimately become resectable. Both the RECIST and the modified RECIST, which measures the size of contrast enhancing portions of lesions, have attempted to correlate pathologic response to radiologic appearance on post-chemotherapy CT scans. As discussed earlier, PET-CT is still being debated and refined to further assist with this issue. Recently, neither the RECIST guidelines nor CT imaging were able to predict the residual tumor burden in those undergoing hepatic resection. ${ }^{55}$ Thus, it is important to note that radiologic response does not yet equate with pathologic response for metastatic disease either, and consequently a resection must include the original sites of liver metastatic disease. ${ }^{56}$ If there is still extra-hepatic disease on subsequent imaging, one must be cautious about proceeding with a hepatic resection, as patients still with regional nodal metastatic disease after neoadjuvant chemotherapy have upwards of a 95\% recurrence rate after hepatic resection at a median time of only 9 months. ${ }^{53}$ That said, multiple and repeated liver resections for recurrent liver metastases can certainly be safely performed..$^{57}$

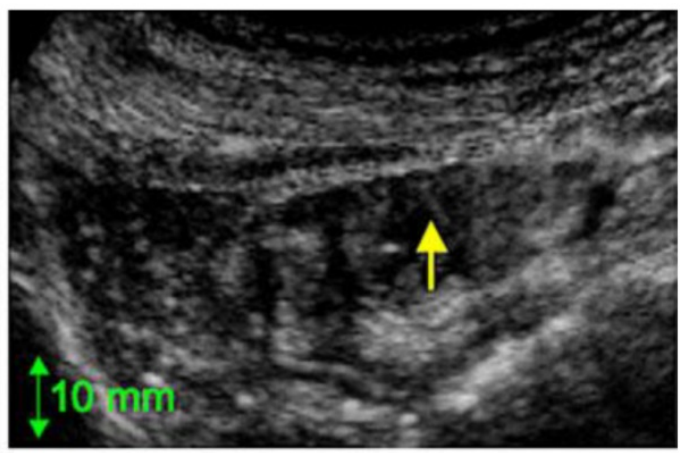

b Regular structure

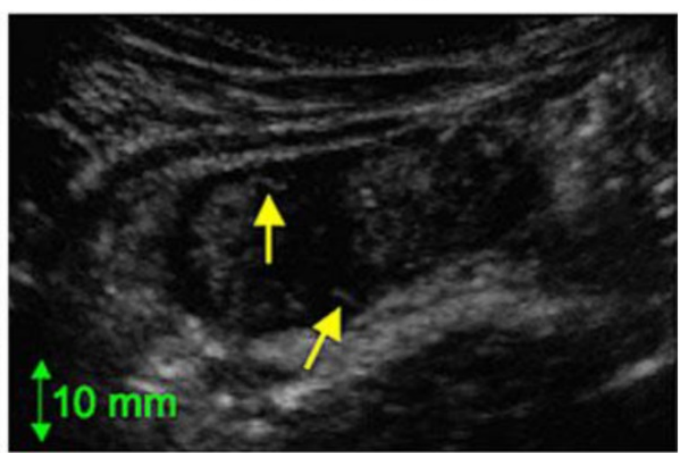

d Vessel diameter $<2 \mathbf{m m}$

Figure 7. Dynamic Contrast US. (a) Irregular structure. Note the twisted blood vessel (arrow). (b) Regular structure. Note the smooth, regular blood vessel (arrow). (c) Diameter of the vessel (arrow) is $>2 \mathrm{~mm}$. (d) Diameter of the vessels (arrows) is $<2 \mathrm{~mm}$. Courtesy of Onji, et al. ${ }^{51}$ 
There are some emerging ways to assess for metastatic response to treatment along with the imaging modalities described above. Higher expression of the gene ABCG2 (ATP-binding cassette sub-family G) has been shown to be associated with metastatic disease and with a lack of response to FOLFOX, while a majority of tumors with low ABCG2 expression responded to FOLFOX. ${ }^{44}$ Circulating tumor cells have been measured within blood samples to predict short-term response to chemotherapy. In a small patient series $(n=14)$ with short-term follow-up, those without any detectable circulating tumor cells after initial chemotherapy lived for at least 1 year and progressed to additional chemotherapy or hepatic metastatic disease resection, while those with circulating tumor cells died within 8 months. ${ }^{58}$ Calculating the numbers of circulating tumor cells during treatment for metastatic CRC may be another adjunct in the ability to determine tumor response.

\section{Watch and Wait: The Next Step for Select Rectal Cancers?}

Radical surgery is still considered fundamental in the treatment of colon and rectal cancer and necessary by many regardless of the tumor response to neoadjuvant CRT. Habr-Gama and associates have shown that in the setting of complete tumor regression after neoadjuvant CRT and an 8 week hiatus, patients with no residual cancer may have a chance to be spared a major surgical procedure. ${ }^{12}$ One key aspect to this approach is the extremely close interval follow-up that is a requirement for these patients. Their algorithm (Figure 2) ${ }^{12}$ includes monthly follow-up visits with DRE and rigid proctoscopy at every visit for the first 3 months and every two to three months during the rest of the first year. CEA levels are drawn every 2 months. Radiological studies, including pelvic CT scans or magnetic resonance imaging, are performed at the time of initial tumor response assessment, and then every 6 months if there are no signs of tumor recurrence. Again, the main objective of these radiological studies is to rule out any sign of residual extra-rectal disease, such as residual nodal disease that would require further investigation or even radical resection. They report a $26.8 \%$ complete clinical response to CRT, avoiding surgery in the majority of these cases, with no apparent detrimental effect on those who subsequently required surgery. ${ }^{12}$ In comparison, a similar study by Dalton and colleagues identified $12 \%$ of patients having CRT demonstrated $\mathrm{CCR}$ that was managed without surgery. A similar proportion of those who had an operation were also found to have had a pCR. This study showed that approximately $24 \%$ of patients were potentially considered complete respond- ers, further adding to the reality of a watch and wait strategy. ${ }^{59}$

This approach is currently being debated in the literature and the question that now arises is whether this is applicable to metastatic colon cancer. The liver is the most common site of CRC metastasis and upwards of $25 \%$ of patients with colorectal cancer will have liver metastases at time of initial diagnosis. ${ }^{52}$ Modern systemic chemotherapy now has response rates in excess of $50 \%$, and in those that do not undergo resection, the median survival approaches 2 years. ${ }^{60}$ In a recent study by Adam and associates, the pCR of liver metastases was associated with a 5-year overall survival of $76 \%{ }^{61}$ In clinical practice, an important open debate is the importance of confirming a histological diagnosis of a cCR. Currently it is generally agreed upon that a positive surgical margin is the only independent predictor of survival besides pathologic response, emphasizing the importance of complete surgical resection even in the era of effective systemic chemotherapy. Advances in imaging must occur and studies proving their superiority to resection will be needed before a watch and wait protocol can be devised for colon cancer metastases.

A watch and wait protocol also has some promising and practice-changing implications for rectal cancer to include alternative treatment options to the current standard of total mesorectal resection or abdominoperineal excision. Patients without residual cancer may be spared from a major surgical procedure and avoid the morbidity that comes with such a procedure to include a temporary/permanent stoma, sexual dysfunction, and fecal incontinence, to name a few. While patients with residual disease may have surgery postponed or delayed without oncological compromise. With regard to colon cancer, and metastatic disease, the verdict is still pending and future studies will need to be performed.

\section{Conclusion}

The management of metastatic colon cancer and locally advanced rectal cancer is changing. Assessment of tumor response is an integral part of this endeavor and plays a pivotal role in the selection of those that will undergo radical surgery and those that should not. The role of chemotherapy regimens, genomic databases/biomarkers, imaging modalities, and surgical techniques are the most widely discussed topics in the literature right now. While a watch and wait approach cannot currently be encouraged outside of a clinical trial, we must continue to push for novel ideas and techniques with the safety of the patient in mind. There is great hope that these innovations will soon be at the disposable of those physicians who treat these patients on a daily basis. 


\section{Disclaimers}

The results and opinions expressed in this article are those of the authors, and do not reflect the opinions or official policy of the United States Army or the Department of Defense.

\section{Competing Interests}

The authors have declared that no competing interest exists.

\section{References}

1. Chang GJ, Kaiser AM, Mills S, Rafferty JF, Buie WD. Practice parameters for the management of colon cancer. Dis Colon Rectum. 2012;55(8):831-843.

2. de Campos-Lobato LF, Stocchi L, da Luz Moreira A, et al. Pathologic complete response after neoadjuvant treatment for rectal cancer decreases distant recurrence and could eradicate local recurrence. Ann Surg Oncol. 2011;18(6):1590-1598.

3. Habr-Gama A, Gama-Rodrigues J, Perez RO. Is tailoring treatment of rectal cancer the only true benefit of long-course neoadjuvant chemoradiation? Dis Colon Rectum. 2013;56(2):264-266.

4. Quah HM, Chou JF, Gonen M, et al. Pathologic stage is most prognostic of disease-free survival in locally advanced rectal cancer patients after preoperative chemoradiation. Cancer. 2008;113(1):57-64.

5. Therasse P, Arbuck SG, Eisenhauer EA, et al. New guidelines to evaluate the response to treatment in solid tumors. European Organization for Research and Treatment of Cancer, National Cancer Institute of the United States, National Cancer Institute of Canada. J Natl Cancer Inst. 2000;92(3):205-216.

6. Sauer R, Becker H, Hohenberger W, et al. Preoperative versus postoperative chemoradiotherapy for rectal cancer. N Engl J Med. 2004;351(17):1731-1740.

7. Dworak O, Keilholz L, Hoffmann A. Pathological features of rectal cancer after preoperative radiochemotherapy. Int J Colorectal Dis. 1997;12(1):19-23.

8. Chari RS, Tyler DS, Anscher MS, et al. Preoperative radiation and chemotherapy in the treatment of adenocarcinoma of the rectum. Ann Surg. 1995;221(6):778-786; discussion 786-777.

9. Seong J, Cho JH, Kim NK, Min JS, Suh CO. Preoperative chemoradiotherapy with oral doxifluridine plus low-dose oral leucovorin in unresectable primary rectal cancer. Int J Radiat Oncol Biol Phys. 2001;50(2):435-439.

10. Nair RM, Siegel EM, Chen DT, et al. Long-term results of transanal excision after neoadjuvant chemoradiation for T2 and T3 adenocarcinomas of the rectum. J Gastrointest Surg. 2008;12(10):1797-1805; discussion 1805-1796.

11. Issa N, Murninkas A, Powsner E, Dreznick Z. Long-term outcome of local excision after complete pathological response to neoadjuvant chemoradiation therapy for rectal cancer. World J Surg. 2012;36(10):2481-2487.

12. Habr-Gama A, Perez RO, Wynn G, Marks J, Kessler H, Gama-Rodrigues J. Complete clinical response after neoadjuvant chemoradiation therapy for distal rectal cancer: characterization of clinical and endoscopic findings for standardization. Dis Colon Rectum. 2010;53(12):1692-1698.

13. Perez RO, Habr-Gama A, Lynn PB, et al. Transanal endoscopic microsurgery for residual rectal cancer (ypT0-2) following neoadjuvant chemoradiation therapy: another word of caution. Dis Colon Rectum. 2013;56(1):6-13.

14. Hughes R, Harrison M, Glynne-Jones R. Could a wait and see policy be justified in T3/4 rectal cancers after chemo-radiotherapy? Acta Oncol. 2010;49(3):378-381

15. Nyasavajjala SM, Shaw AG, Khan AQ, Brown SR, Lund JN. Neoadjuvant chemo-radiotherapy and rectal cancer: can the UK watch and wait with Brazil? Colorectal Dis. 2010;12(1):33-36.

16. Hiotis SP, Weber SM, Cohen AM, et al. Assessing the predictive value of clinical complete response to neoadjuvant therapy for rectal cancer: an analysis of 488 patients. I Am Coll Surg. 2002;194(2):131-135; discussion 135-136

17. Hayden DM, Jakate S, Pinzon MC, et al. Tumor scatter after neoadjuvant therapy for rectal cancer: are we dealing with an invisible margin? Dis Colon Rectum. 2012;55(12):1206-1212.

18. Berman JM, Cheung RJ, Weinberg DS. Surveillance after colorectal cancer resection. Lancet. 2000;355(9201):395-399.

19. Park YA, Lee KY, Kim NK, Baik SH, Sohn SK, Cho CW. Prognostic effect of perioperative change of serum carcinoembryonic antigen level: a useful tool for detection of systemic recurrence in rectal cancer. Ann Surg Oncol. 2006;13(5):645-650.

20. Park YJ, Park KJ, Park JG, Lee KU, Choe KJ, Kim JP. Prognostic factors in 2230 Korean colorectal cancer patients: analysis of consecutively operated cases. World J Surg. 1999;23(7):721-726.

21. Thirunavukarasu $\mathrm{P}$, Sukumar $\mathrm{S}$, Sathaiah $\mathrm{M}$, et al. C-stage in colon cancer: implications of carcinoembryonic antigen biomarker in staging, prognosis, and management. I Natl Cancer Inst. 2011;103(8):689-697.

22. Jang NY, Kang SB, Kim DW, et al. The role of carcinoembryonic antigen after neoadjuvant chemoradiotherapy in patients with rectal cancer. Dis Colon Rectum. 2011;54(2):245-252.
23. Bos JL, Fearon ER, Hamilton SR, et al. Prevalence of ras gene mutations in human colorectal cancers. Nature. 1987;327(6120):293-297.

24. Richman SD, Seymour MT, Chambers P, et al. KRAS and BRAF mutations in advanced colorectal cancer are associated with poor prognosis but do not preclude benefit from oxaliplatin or irinotecan: results from the MRC FOCUS trial. J Clin Oncol. 2009;27(35):5931-5937.

25. Van Cutsem $\mathrm{E}$, Kohne $\mathrm{CH}$, Lang $\mathrm{I}$, et al Cetuximab plus irinotecan, fluorouracil, and leucovorin as first-line treatment for metastatic colorectal cancer: updated analysis of overall survival according to tumor KRAS and BRAF mutation status. J Clin Oncol. 2011;29(15):2011-2019.

26. Wang L, Cunningham JM, Winters JL, et al. BRAF mutations in colon cancer are not likely attributable to defective DNA mismatch repair. Cancer Res. 2003;63(17):5209-5212.

27. Souglakos J, Philips J, Wang R, et al. Prognostic and predictive value of common mutations for treatment response and survival in patients with metastatic colorectal cancer. Br J Cancer. 2009;101(3):465-472.

28. Pakneshan S, Salajegheh A, Smith RA, Lam AK. Clinicopathological relevance of BRAF mutations in human cancer. Pathology. 2013;45(4):346-356.

29. Muller HM, Oberwalder M, Fiegl H, et al. Methylation changes in faecal DNA: a marker for colorectal cancer screening? Lancet. 2004;363(9417):1283-1285.

30. Toyota M, Ahuja N, Ohe-Toyota M, Herman JG, Baylin SB, Issa JP. CpG island methylator phenotype in colorectal cancer. Proc Natl Acad Sci U S A. 1999;96(15):8681-8686.

31. Weisenberger DJ, Siegmund KD, Campan M, et al. CpG island methylator phenotype underlies sporadic microsatellite instability and is tightly associated with BRAF mutation in colorectal cancer. Nat Genet. 2006;38(7):787-793

32. Desch $\mathrm{CE}$, Benson $\mathrm{AB}$, 3rd, Somerfield $\mathrm{MR}$, et al. Colorectal cancer surveillance: 2005 update of an American Society of Clinical Oncology practice guideline. J Clin Oncol. 2005;23(33):8512-8519.

33. Bipat S, van Leeuwen MS, Comans EF, et al. Colorectal liver metastases: CT, MR imaging, and PET for diagnosis--meta-analysis. Radiology. 2005;237(1):123-131.

34. Thoeni RF, Rogalla P. CT for the evaluation of carcinomas in the colon and rectum. Semin Ultrasound CT MR. 1995;16(2):112-126.

35. Schaefer O, Langer M. Detection of recurrent rectal cancer with CT, MRI and PET/CT. Eur Radiol. 2007;17(8):2044-2054.

36. Chun YS, Vauthey JN, Boonsirikamchai P, et al. Association of computed tomography morphologic criteria with pathologic response and survival in patients treated with bevacizumab for colorectal liver metastases. JAMA. 2009;302(21):2338-2344.

37. Ricotta R, Vanzulli A, Moroni M, et al. Magnetic resonance imaging as an early indicator of clinical outcome in patients with metastatic colorectal carcinoma treated with cetuximab or panitumumab. Clin Colorectal Cancer. 2013;12(1):45-53.

38. Koh DM, Blackledge M, Padhani AR, et al. Whole-body diffusion-weighted MRI: tips, tricks, and pitfalls. AJR Am J Roentgenol. 2012;199(2):252-262.

39. Lambregts DM, Vandecaveye V, Barbaro B, et al. Diffusion-weighted MRI for selection of complete responders after chemoradiation for locally advanced rectal cancer: a multicenter study. Ann Surg Oncol. 2011;18(8):2224-2231.

40. Miles KA, Griffiths MR. Perfusion CT: a worthwhile enhancement? Br J Radiol. 2003:76(904):220-231.

41. Perez RO, Habr-Gama A, Sao Juliao GP, et al. Clinical Relevance of PET/CT positive inguinal nodes in rectal cancer after neoadjuvant chemoradiation. Colorectal Dis. 2013 Jun;15(6):674-82

42. Ptok H, Marusch F, Meyer F, et al. Feasibility and accuracy of TRUS in the pre-treatment staging for rectal carcinoma in general practice. Eur J Surg Oncol. 2006;32(4):420-425.

43. Rieger N, Tjandra J, Solomon M. Endoanal and endorectal ultrasound: applications in colorectal surgery. ANZ J Surg. 2004;74(8):671-675.

44. Gerdes B, Langer P, Kopp I, Bartsch D, Stinner B. Localization of the peritoneal reflection in the pelvis by endorectal ultrasound. Surg Endosc. 1998;12(12):1401-1404

45. Gavioli M, Bagni A, Piccagli I, Fundaro S, Natalini G. Usefulness of endorectal ultrasound after preoperative radiotherapy in rectal cancer: comparison between sonographic and histopathologic changes. Dis Colon Rectum. 2000;43(8):1075-1083

46. Radovanovic Z, Breberina M, Petrovic T, Golubovic A, Radovanovic D. Accuracy of endorectal ultrasonography in staging locally advanced rectal cancer after preoperative chemoradiation. Surg Endosc. 2008;22(11):2412-2415.

47. Cartana ET, Parvu D, Saftoiu A. Endoscopic ultrasound: current role and future perspectives in managing rectal cancer patients. I Gastrointestin Liver Dis. 2011;20(4):407-413

48. Kim JC, Kim HC, Yu CS, et al. Efficacy of 3-dimensional endorectal ultrasonography compared with conventional ultrasonography and computed tomography in preoperative rectal cancer staging. Am I Surg. 2006;192(1):89-97.

49. Giovannini M, Bories E, Pesenti C, Moutardier V, Lelong B, Delpero JR. Three-dimensional endorectal ultrasound using a new freehand software program: results in 35 patients with rectal cancer. Endoscopy. 2006;38(4):339-343

50. Lassau N, Chami L, Benatsou B, Peronneau P, Roche A. Dynamic contrast-enhanced ultrasonography (DCE-US) with quantification of tumor perfusion: a new diagnostic tool to evaluate the early effects of antiangiogenic treatment. Eur Radiol. 2007;17 Suppl 6:F89-98. 
51. Onji K, Yoshida S, Tanaka S, et al. Microvascular structure and perfusion imaging of colon cancer by means of contrast-enhanced ultrasonography. Abdom Imaging. 2012;37(2):297-303.

52. Ochiai T, Masuda T, Yagi M, et al. Successful Combination Therapy of Radical Liver Resection With 5-Fluorouracil/leucovorin, Oxaliplatin, Plus Bevacizumab for Ascending Colon Cancer With Pulmonary and 43 Liver Metastases: Report of a Case. Int Surg. 2012;97(1):6-13.

53. Wanebo HJ, LeGolvan M, Paty PB, et al. Meeting the biologic challenge of colorectal metastases. Clin Exp Metastasis. 2012;29(7):821-839.

54. Hsu YN, Lin JK, Chen WS, et al. A new classification scheme for recurrent or metastatic colon cancer after liver metastasectomy. I Chin Med Assoc. 2011;74(11):493-499.

55. Egger ME, Cannon RM, Metzger TL, et al. Assessment of chemotherapy response in colorectal liver metastases in patients undergoing hepatic resection and the correlation to pathologic residual viable tumor. J Am Coll Surg. 2013;216(4):845-856; discussion 856-847.

56. Adams RB, Aloia TA, Loyer E, Pawlik TM, Taouli B, Vauthey JN. Selection for hepatic resection of colorectal liver metastases: expert consensus statement. HPB (Oxford). 2013;15(2):91-103.

57. Takahashi S, Nagai K, Saito N, et al. Multiple resections for hepatic and pulmonary metastases of colorectal carcinoma. Jpn J Clin Oncol. 2007;37(3):186-192

58. Neki K, Kawahara H, Watanabe K, Toyama Y, Akiba T, Yanaga K. Usefulness of circulating tumor cells after preliminary chemotherapy for prediction of response to further anticancer therapy in patients with initially unresectable metastatic colorectal cancer. Anticancer Res. 2013;33(4):1769-1772.

59. Dalton RS, Velineni R, Osborne ME, et al. A single-centre experience of chemoradiotherapy for rectal cancer: is there potential for nonoperative management? Colorectal Dis. 2012;14(5):567-571.

60. Catenacci DV, Kozloff M, Kindler HL, Polite B. Personalized colon cancer care in 2010. Semin Oncol. 2011;38(2):284-308.

61. Adam R, Avisar E, Ariche A, et al. Five-year survival following hepatic resection after neoadjuvant therapy for nonresectable colorectal. Ann Surg Oncol. 2001;8(4):347-353.

62. Eisenhauer EA, Therasse P, Bogaerts J, et al. New response evaluation criteria in solid tumours: revised RECIST guideline (version 1.1). Eur J Cancer. 2009;45(2):228-247.

63. Engin G, Sharifov R, Gural Z, et al. Can diffusion-weighted MRI determine complete responders after neoadjuvant chemoradiation for locally advanced rectal cancer? Diagn Interv Radiol. 2012;18(6):574-581. 